\title{
The Effectiveness of Kinesio-Taping on Pain and Quality of Life in Participants with Lower Abdominal Surgery
}

\author{
Vaishali D. Suthar ${ }^{1}$, V. P. Hathila ${ }^{2}$ \\ ${ }^{1}$ Assistant Professor, Ph.D. Scholar, Ahmedabad Institute of Medical Sciences (Affiliated to Gujarat University), \\ Ahmedabad, Gujarat, India. \\ ${ }^{2}$ General Surgeon and Dean of Medical Faculty, Parul Institute of Medical Sciences (Affiliated to Parul \\ University) Vadodara, Gujarat, India. \\ Corresponding Author: Vaishali D. Suthar
}

\begin{abstract}
Purpose: Many studies in physiotherapy using kinesio-tape are available to prevent and cure the musculoskeletal and neurological disorders in patients. However, few studies are available for using Kinesio-tape in abdominal surgery patients. Therefore, the need arises to assess the effectiveness of Kinesio-tape on pain and quality of life in participants with lower abdominal surgery. The secondary objectives are to assess the effectiveness of Kinesio-taping in improving patient outcomes in form of pain relief and ambulation as well the quality of life and to determine the level of prescribing of Kinesio-taping immediately after abdominal surgery.
\end{abstract}

Relevance: The present study helps to manage post surgical patients providing quality life and faster recovery, less usage of analgesic drugs.

Participants: Total 78 participants with age between 18years to 65 years old both males and females underwent lower abdominal surgery allocated randomly.

Methods: The participants were divided into two groups: Control group received conventional treatment and experimental group received conventional treatment and Kinesio-taping. Following assessment tools were used as outcome measure in both the groups and was administered 4 hours of surgery. Numerical Pain Rating Scale (NPRS), Abdominal Circumference (AC) and Post-operative Quality of Recovery 15 score (QoR 15). NPRS was done pre and post treatment on the basis of first 24hrs, 48hrs till $5^{\text {th }}$ day post-operatively. AC was measured post treatment on the basis of first 24hrs, $48 \mathrm{hrs}$ till $5^{\text {th }}$ day post-operatively. QoR-15 was taken post treatment on $2^{\text {nd }}$ and $4^{\text {th }}$ day.

Analysis: Descriptive statistics and data was analysed on SPSS by adopting proper statistical tests i.e. Mann-Whitney test and one way ANOVA.

Results: There was significant difference found between control and experimental group in all three outcome measures.

Conclusion: There was faster recovery with kinesio-tape application in lower abdominal surgery participants.

Implications: It is recommended to apply kinesio-tape with physiotherapy in post abdominal surgery.

Key Words: Pain, Kinesio-tape, Abdominal surgery

\section{INTRODUCTION}

The complications which occur after surgical operations is connected with malfunction or failure of the alimentary canal. This malfunction may be the cause of flatulence and oedema of the stomach cover, which leads to pain. The other possible reason for post surgical complication is post-operative immobilization which is caused by hypokinesis ${ }^{1}$.

Physiotherapy in patients after surgeries should lead to the fastest possible 
mobilization of the patient and the time of recovery and return to normal physical activity depends on the extent of surgical operation and patient's condition.

The advanced technique known as kinesio-taping in post abdominal surgery suggest its usefulness for treatment of pain, accelerating post-operative wound healing processes. It is a therapeutic technique which was developed by Dr. Kenzo Kase in Japan more than 25 years before. Kinesiotaping applications individually suited to patients' needs, support, curing processes and provide new quality of physiotherapy.

According to the manufacturers of Kinesio-taping, the tape causes microconvolutions or folds in the skin which causes a lifting of the skin away from the tissue beneath. This facilitates a release in pressure on tender tissues underneath and provides space for lymphatic fluid movement, increases circulation of blood which helps to fasten wound healing. This can help relieve pain, prevent over contraction, facilitate lymphatic drainage and improve kinesthetic awareness ${ }^{2}$.

Kinesio-taping can be applied in four basic therapeutic aims: Mechanical correction which gives stabilization, muscular and fascial tone normalization, improvement and correction of mobility range, pain and swelling reduction. Restoration of normal fluid perfusion helps in facilitation of lymph blood flow, swelling reduction and resulting reduction of incorrect sensibility and pain of skin and muscles. Provides support for muscular activity to relax muscles, fatigue reduction and restoration of mobility range and pain relief. Analgesic system activation helps in elimination of pain cause and activation of pain inhibitors ${ }^{3}$.

Many studies in physiotherapy using kinesio-tape are available to prevent and cure the musculoskeletal and neurological disorders in patients. But few studies are available for using kinesio-tape in abdominal surgery patients.

Therefore, need arise to assess the effectiveness of kinesio-tape on pain and quality of life in participants with lower abdominal surgery.

\section{REVIEW OF THE LITERATURE Lower abdominal surgery review: Post operative pain:}

All low abdominal incisions entailed a less statistically significantly partial oxygen pressure and carbon dioxide, less pulmonary shunt increase, less pain and less use of analgesic than upper abdominal incisions. Upper abdominal incisions caused substantial respiratory impairments and disturbances in terms of hypoxia, hyperventilation and pulmonary shunt increase ${ }^{4}$.

Many studies concluded that postoperative pain is one of the most common therapeutic problems in hospitals ${ }^{5}$. It can increase morbidity, leading to reduced breathing and cough suppression, facilitating retained pulmonary secretions and pneumonia ${ }^{6}$ and delaying normal gastric and bowel function, thus contributing to a longer recovery period ${ }^{7,8}$.

\section{Kinesio-taping review:}

In post abdominal surgery, Kinesiotaping has a significant reduction in pain perception, increase the tolerance effort and improve the patient's total experience. This gives a support for kinesio-taping in postoperative pain ${ }^{1}$.

Highly effective in treating patients after abdominal surgery. The daily decrease of $2.5 \%$ points in the abdomen circumference helped to relieve pain and reduces doses of pain killers. Within the control group, after the initial increase in the abdomen circumference measurement values, they finally decreased by $0.5 \%$ points on average ${ }^{3}$.

Kinesio-taping could be applied as another choice of myofascial trigger pain therapy ${ }^{9}$.

The clinical efficacy of Kinesiotaping for shoulder pain proved that patients can wear the tape even in the shower or pool without the need for reapplication as the tape is latex-free and features an adhesive 
that is $100 \%$ heat activated acrylic. The $100 \%$ cotton fibers allow for evaporation and fast drying, thereby ensuring that patients can wear the tape even in the shower or pool without the need for reapplication. This allows for a wear time of 3-5 days and makes the treatment more economical ${ }^{10,11}$.

Kinesio-tape applied over lower trunk may increase active lower trunk flexion ROM. Improvements in ROM were attributed to both increased blood circulation and stimulation of cutaneous mechano-receptors at the taped area ${ }^{12}$.

Clinical therapeutic applications of the kinesio-taping method have proposed several benefits depending on the amount of stretch applied to the tape during application, to provide positional stimulus through the skin, to align fascial tissues, to create more space by lifting fascia and soft tissues above the area of pain/inflammation, to provide sensory stimulation to assist or limit motion, to assist in the removal of oedema by directing exudates toward a lymph duct ${ }^{13}$.

Kinesio-taping seems to be a safe and promising approach to reduce postoperative pain and the dose of analgesic drugs to be administered after cardiac surgery via sternotomy ${ }^{14}$.

The research shows the efficiency of kinesio-taping employed for physiotherapy of patients after laparoscopic cholecystectomy. Kinesio-taping provides effective support for physiotherapy and through post-operative wound stabilization reduces functional activity disorders resulting from Cholecystectomy allowing for shortening of hospitalization time ${ }^{21}$.

\section{Hypothesis:}

Null hypothesis(H0): No significant changes in outcome measures between the control and experimental group.

Alternate hypothesis(H1): Significant changes in outcome measures between the control and experimental group.

\section{AIM \& OBJECTIVES:}

AIM: To find out the effectiveness of Kinesio-taping on pain and quality of life in subjects with lower abdominal surgery.

\section{OBJECTIVES:}

1. To study the effects of kinesio-taping in post abdominal surgery.

2. To compare the effects of kinesio-taping between control and experimental group.

3. To assess the effectiveness of kinesiotaping in improving patient outcomes in form of pain relief and ambulation as well the quality of life.

4. To determine the level of prescribing of kinesio-taping immediately after abdominal surgery.

\section{Problem Statement / Justification:}

In post abdominal surgery exclusive effect of kinesio-taping is difficult to predict.

Need to differentiate the faster recovery in both the groups.

Expert application of kinesio-taping is mandatory.

\section{MATERIALS AND MATHODS}

Materials: Kinesio-tape roll, Scissor, measure tape, pen.

Source of data: From Surgical and maternity hospitals, Ahmedabad.

Method of data collection: Convenient and allocation of group by randomization of participants.

\section{Inclusion criteria:}

- Age between 18-65 years old both male and female, underwent lower abdominal surgery.

\section{Exclusion criteria:}

- Upper abdominal surgery

- Laparoscopy surgery

- Severe cardiac and lung conditions such as myocardial infarction, atelectasis, $\mathrm{k} / \mathrm{c} / \mathrm{o}$ diabetes and post-operative complications (e.g. fever, wound infection), neurological impairment. 


\section{Study design: Randomized controlled clinical trial}

Before application of Kinesiotaping, skin sensitivity test was done preoperatively to check any allergic reaction due to Kinesio-taping.

Skin sensitivity test for Kinesio-taping: A piece of kinesio-tape was applied over flexor aspect of forearm for 6-8 hours with stretch of inhibitory technique. Skin reaction by means of itching, redness was noted in randomly selected participants.

The participants were randomly divided into two groups.

Group 1- Control group (40 participants) only conventional treatment given

Group 2- Experimental group (38 participants) conventional treatment followed by Kinesio-tape

Sample size: 78

Assessment criteria: Basic demographic information was collected and baseline evaluations were performed at the time of post-operative to assess the primary (physical function) and secondary (pain and pulmonary function) dependent variables. Both the groups were received standard methods of treatment by means of medical care and physiotherapy.

The participants consents were obtained to participate in the study. All the volunteered participants were randomly divided into two groups. The control group and the experimental group.

Following assessment tools were used as outcome measures in both the groups and was administered after 4 hours of surgery as the participant gets withdrawn from anaesthetic effect.

1. Numerical Pain Rating Scale (NPRS)

2. Abdominal Circumference (AC)

3. Post-operative Quality of Recovery 15 score (QoR 15)

The outcome measure NPRS was done pre and post treatment on the basis of first 24hrs, 48hrs, $72 \mathrm{hrs} . .$. till $5^{\text {th }}$ day postoperatively. The Abdominal Circumference was measured immediately after treatment on the basis of first 24hrs, 48hrs, 72hrs...till $5^{\text {th }}$ day postoperatively. The QoR-15 was taken immediately after treatment on $2^{\text {nd }}$ and $4^{\text {th }}$ day postoperatively.

In group 1 (Control group): Participants received conventional treatment by means of medical care and physiotherapy. Physiotherapy care included education about post-operative complications, bed exercises (hip, knee and ankle movements), early transfer to chair (on day of surgery or POD1) and ambulation, diaphragmatic breathing and manual techniques as clinically indicated. All patients were seen by a physiotherapist once a day for approximately 20min. Informed consent form was delivered to each participant prior to procedure. NPRS was assessed prior to conventional treatment and immediately after conventional treatment till $5^{\text {th }}$ day. Abdominal Circumference (AC) was assessed immediately after the conventional treatment till $5^{\text {th }}$ day. QoR 15 was assessed on second and fourth day immediately after treatment.

In group 2 (Experimental group): Participants received conventional treatment and Kinesio-tape applications. A clear explanation about the kinesio-tape procedure was given to each participant, that was applied post-operatively after the conventional treatment and was changed every 24 hours until $5^{\text {th }}$ day post-operative in addition to the standard post-operative care.

\section{Kinesio tape application:}

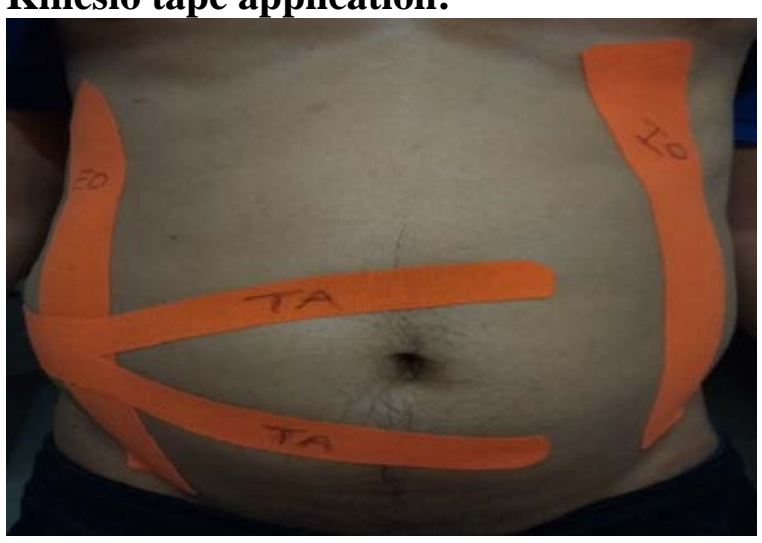




\section{Kinesio tape application on subject with LSCS}

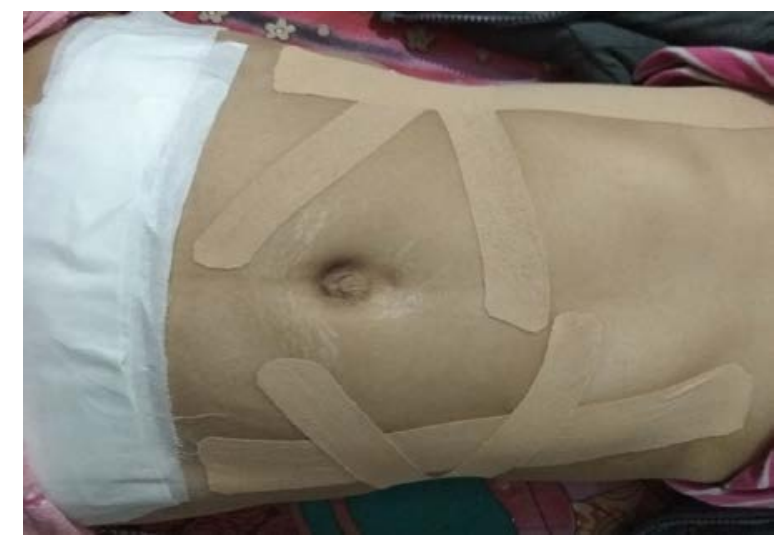

Following kinesio-taping techniques were used over abdominal area.

Muscle inhibition application: Kinesiotaping was applied from distal to proximal attachment on external oblique (EO) muscle on the left side with $15 \%$ of stretch. This technique is more preferable in very acute stage as mechanically it inhibits and relaxes the muscle and thus inhibits pain.

Muscle facilitation application: Kinesiotaping was applied from proximal to distal attachment on internal oblique (IO) muscle on right side with $25 \%$ of stretch. For transversus abdominis (TA) muscle Kinesio-tape was applied proximal to distal attachment of bilaterally with $25 \%$ of stretch. During the application patient was instructed to inhale in his/her comfort zone to stimulate the stretch reflex. Kinesio-tape was applied for 24 hours. Informed consent form was delivered to each participant prior to procedure.

NPRS was assessed prior to conventional and Kinesio-tape application and immediately after conventional and Kinesio-tape application till $5^{\text {th }}$ day.

Abdominal Circumference (AC) was assessed immediately after the conventional and kinesio-tape application till $5^{\text {th }}$ day.

QoR 15 was assessed on second and fourth day immediately after conventional and Kinesio-tape application.
Numerical Pain Rating Scale (NPRS): It is a segmented numeric version of the visual analog scale (VAS) in which a respondent selects a whole number (0-10 integers) that best reflects the intensity of their pain ${ }^{15}$. The common format is a horizontal line or bar ${ }^{16}$. Similar to the pain VAS, the NPRS is anchored by terms describing pain severity extremes. NPRS is a valid test and has testretest reliability in both literate and illiterate patients ${ }^{17}$.

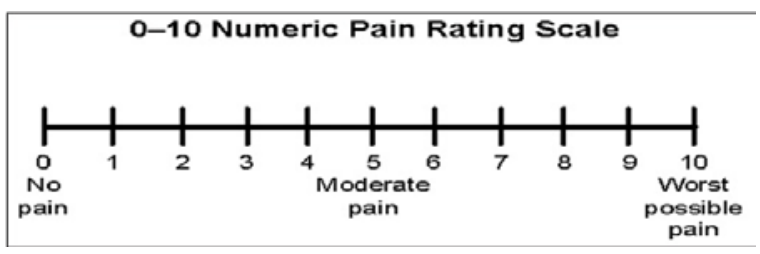

Abdominal Circumference (AC): The measurement of abdominal circumference taken from navel and taken in equal intervals of time, beginning after finishing the first 24 hour period of treatment ${ }^{3}$.

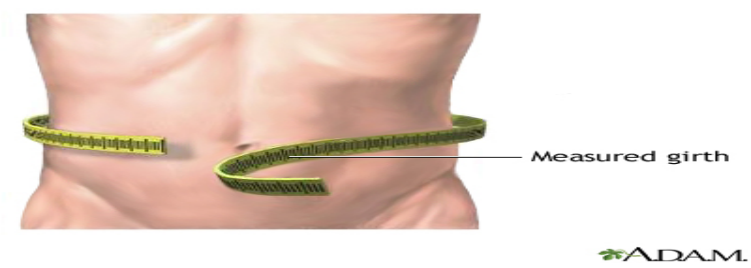

QoR-15: It consists of 15 item patient rated postoperative QoR score, the QoR-15 and provides a valid, reliable, responsive and easy to use method of measuring the quality of a patient's postoperative recovery. The QoR-15 can be a valuable outcome measure in perioperative clinical trials, and for assessing the impact of changes in health care delivery for quality assurance purposes $^{18}$. The validity, reliability, responsiveness and clinical acceptability and feasibility of the score were excellent with most patients able to complete the questionnaire in less than $3 \mathrm{~min}$. it has a score range of 0-150. 


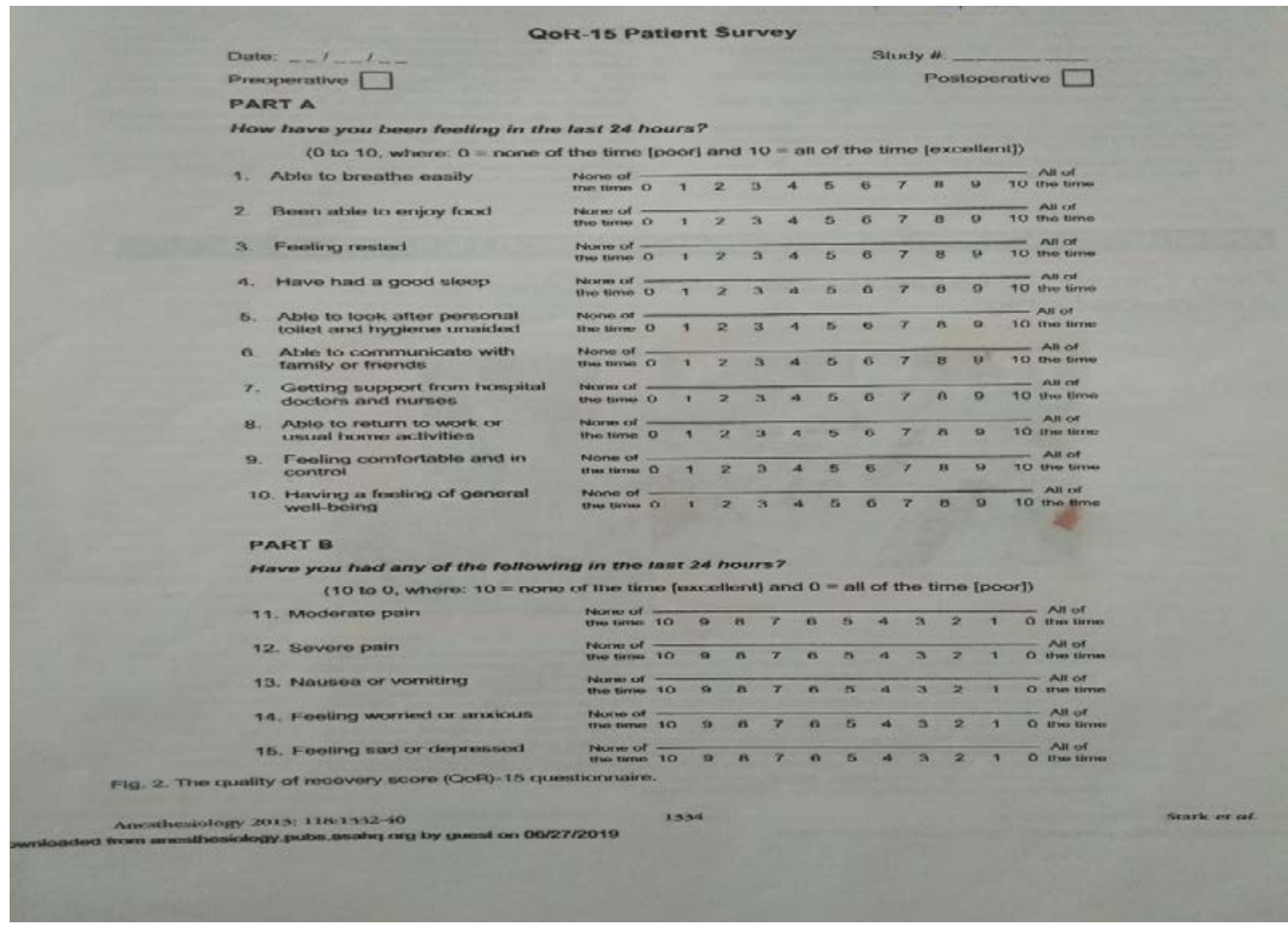

\section{Statistical methods for analysis:}

All the collected data were inserted in the SPSS to apply both descriptive and inferential statistical analysis.

Mann-Whitney test was used to compare the outcomes between groups and one way ANOVA was used to compare the outcomes within groups. The level of significance was at 0.05 .

\section{RESULT}

Total 78 patients (58 females, 20 males) were participated in the current study with mean age (40.83+_5.99). They underwent different lower abdominal surgeries, the experimental group (21 LSCS, 6 abdominal hysterectomy, 10 appendicectomy \& 1 inguinal hernia) and the control group (25 LSCS, 6 abdominal hysterectomy, 7 appendicectomy \& 2 inguinal hernia).

Regarding NPRS, both experimental (6.55 \pm 0.79$)$ and control groups $\left(2.8+\_0.56\right)$ showed between groups a significant difference on NPRS, between pre NPRS D1 and post NPRS D5 $(\mathrm{U}=1.5)(\mathrm{p}<0.001)$.

In addition there was significant difference found in abdominal circumference (AC) between experimental $(6.62+$ 1.24) and control group $\left(0.65+\_0.41\right)$ day 1 and day 5 respectively $(\mathrm{p}<0.001)$.

Furthermore, the PO QoR-15 showed significant difference between experimental (52.97 \pm 7.13$)$ and control $(25.5+8.02)$ groups on $2^{\text {nd }}$ and $4^{\text {th }}$ day $(\mathrm{p}<0.001)$.

Table 1. Gender distribution
\begin{tabular}{|l|l|l|l|}
\hline Gender & Frequency & Percent \\
\hline Group & $\mathrm{F}$ & 27 & 71.1 \\
\cline { 2 - 4 } Experimental Group & $\mathrm{M}$ & 11 & 28.9 \\
\cline { 2 - 4 } & Total & 38 & 100.0 \\
\hline Control Group & $\mathrm{F}$ & 31 & 77.5 \\
\cline { 2 - 4 } & $\mathrm{M}$ & 9 & 22.5 \\
\cline { 2 - 4 } & Total & 40 & 100.0 \\
\hline
\end{tabular}

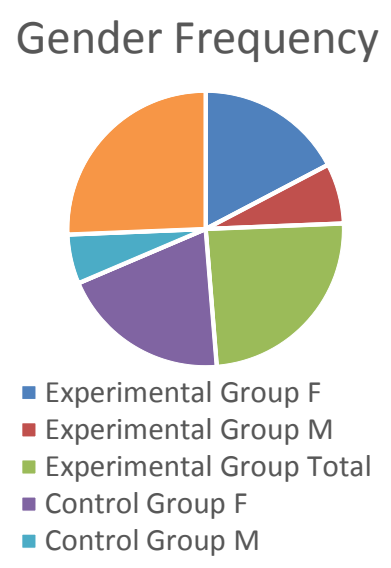


Vaishali D. Suthar et.al. The effectiveness of Kinesio-taping on pain and quality of life in participants with lower abdominal surgery.

Table 2. Difference in NPRS, AC and QoR-15 score in both groups

\begin{tabular}{|l|l|l|l|l|l|l|}
\hline & \multicolumn{2}{|c|}{ Experimental } & \multicolumn{2}{|c|}{ Control } & \multirow{2}{*}{ Whitney } & \multirow{2}{*}{ P Value } \\
\hline & Mean & SD & Mean & SD & & \\
\hline Difference Pre NPRS day1 and Post NPRS day5 & 6.55 & 0.795 & 2.8 & 0.564 & 1.5 & $<0.001$ \\
\hline Difference AC Day1 and Day5 & 6.62 & 1.24 & 0.65 & 0.41 & 0 & $<0.001$ \\
\hline Difference POQOR Day4 to Day2 & 52.97 & 7.13 & 25.5 & 8.02 & 6 & $<0.001$ \\
\hline
\end{tabular}

Bar diagram shows the difference in mean of both groups.

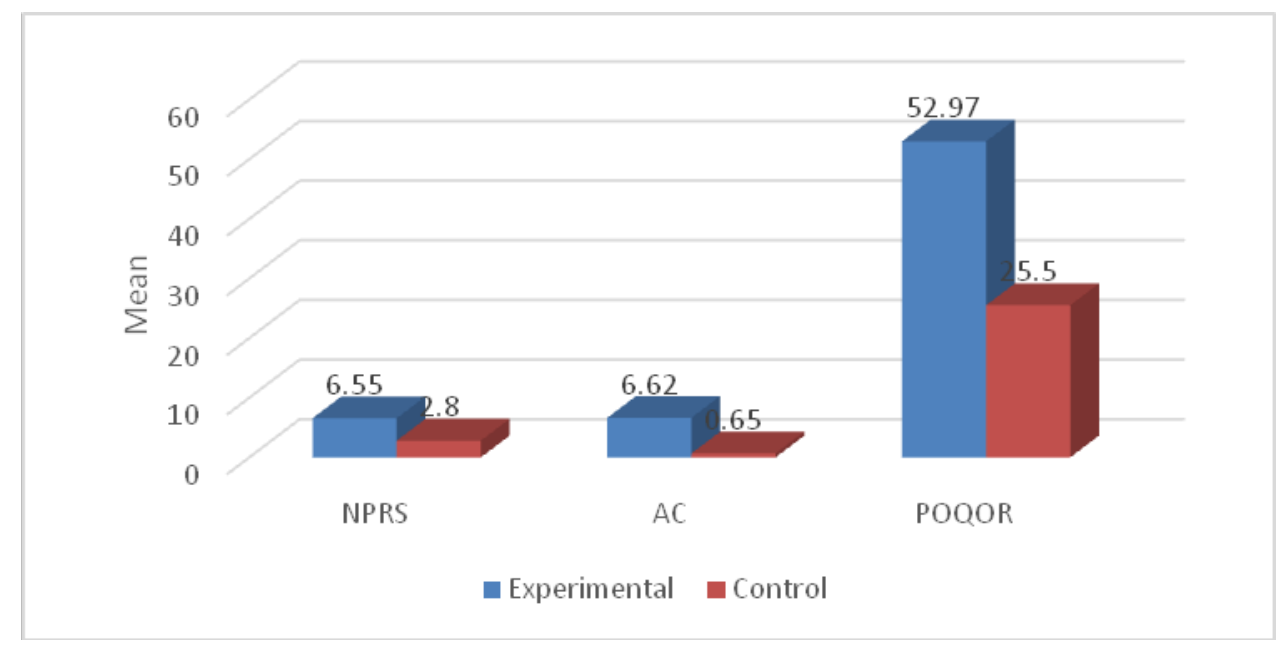

Difference Pre NPRS day1 and Post NPRS day 5

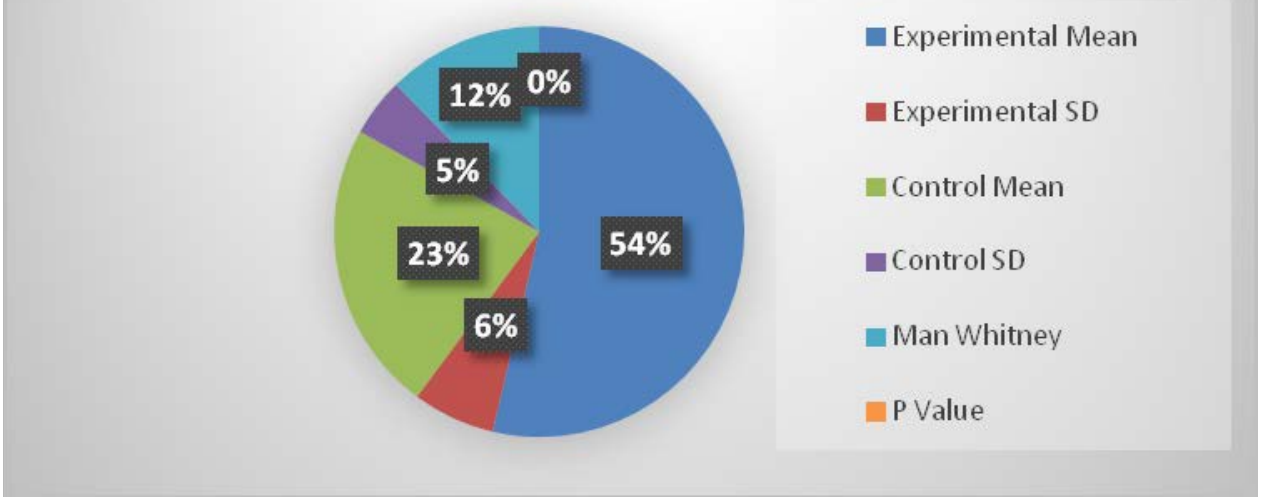

\section{DISCUSSION}

The current study showed significant $(\mathrm{p}<0.001)$ pain reduction in experimental group in NPRS and the possible explanations to this reduction are, Kinesio tape's elasticity creates skin folds which can lift the skin to increase space between skin and muscle to improve circulation of blood and lymph. Since this space contains a variety of nerve receptors that send specific information to the brain. Kinesio tape modulates the information that is sent by the receptors to the brain and makes less reactive responses in the body. This process allows the body to have an adaptive normal function by moving out of the barrier which naturally slow down the recovery process ${ }^{19}$.

In addition, taping activates neurological suppression in order to reduce pain and increase joint range of motion ${ }^{21}$. Also, Kinesio tape lift the fascia and soft tissue above the areas of pain, align fascial tissues and provide positional stimulus through the skin ${ }^{20}$.

Yoshida and Kahanov also stated that Kinesio tape is to decrease pain by stimulating the neuromuscular system; assists in restoration of proper muscle function and realignment of joints as a result of injury or disease. Finally, Kinesio tape 
enhances comprehensive body function by improving the flow of blood and lymphatic fluid throughout the body ${ }^{12}$.

Marcin Krajczy et al concluded that kinesio taping is an effective method of physiotherapy support in patients after abdominal surgery, there was regular and significant reduction was obtained in abdominal circumference what caused pain relief and use of fewer analgesic agents ${ }^{3}$. Another possible theory to be taken into account for the analgesic effects of KT is the gate control theory of pain modulation. The tape has been suggested to stimulate neuromuscular pathways via afferent feedback. Increased afferent stimulus to large diameter nerve fibers might reduce pain perception level due to an input decrease from the small diameter nerve fibers conducting nociception ${ }^{21,22}$.

Thelen et al refer to this theory in their work on KT applications in shoulder pain $^{23}$. Also, Gonz'alez-Iglesias et al. achieve pain- relief effects of KT applications in patients with acute whiplash injury ${ }^{24}$.

Ethical Clearance- Ethical clearance is received from Institutional Ethical Committee for Human Research (PUIECHR). CTRI registration been done. Availability of the patient, their cost analyse for research purpose and time period is done.

Declaration- The prior consent of the participants was taken before application of Kinesio-taping and the work was carried out after getting clearance from the institutional ethics committee.

\section{Acknowledgement: None}

\section{Conflict of Interest: None}

\section{Source of Funding: None}

\section{REFERENCES}

1. Sayed Tantawy, Dalia Kamel, October 2015, Effect of kinesio-taping on pain post laparoscopic abdominal surgery: randomized controlled trial, International Journal of Therapies and Rehabilitation Research. DOI:10.5455/ijtrr.00000098

2. Akbas E, Atay O, Yuksel L. The effects of additional kinesiotaping over exercise in the treatment of patellofemoral pain syndrome. Acta Orthop Traumatol Turc 2011; 45:335341.

3. Marcin Krajczy et al, January 2007, "Kinesiotaping in physiotherapy after abdominal surgery", Fizjoterapia Polska, Vol. 7, 299-307.

4. Zeljko Mimica et al, 2007, "Effect of surgical incision on pain and respiratory function after abdominal surgery: A randomized clinical trial”, HepatoGastroenterology; 54:2216-2220.

5. Donovan M, Dillon P, McGuire L. Incidence and characteristics of pain in a sample of medical-surgical inpatients. Pain 1987; 30: 69-78.

6. Sydow, W. The influence of anesthesia and postoperative analgesic management on lung function. Acta Chir Scand 1989; 550 (Suppl.): 159-165.

7. Wattwil, M. Postoperative pain relief and gastrointestinal motility. Acta Chir Scand 1989; 550 (suppl.): 140-145.

8. Rowbotham D. Postoperative pain. Prescr 1993; 33:237-243.

9. Wei-Ting $\mathrm{Wu}$, Chang-Zern Hong and LiWei Chou, The kinesio-taping method for myofascial pain control. Evidence based complementary and alternative medicine, vol. 2015, article ID 950519, 9 pages.

10. Mehran Mostafavifar, MD, Jess Wertz DO et al, A systematic review of the effectiveness of kinesio taping for musculoskeletal injury, DOI: 10.3810/psm.2012.11.1986.

11. Thelen MD, Dauber JA, Stoneman PD. The clinical efficacy of kinesio tape for shoulder pain: a randomized double-blinded, clinical trial. J Orthop Sports Phys Ther: 2008; 38(7): 389-395.

12. Yoshida, A., \& Kahanov, L. (2007). The effects of kinesio taping in lower trunk range of motions. Research in sports medicine, 15, 103-112.

13. Kase $\mathrm{K}$, Wallis J, Kase T. Clinical therapeutic applications of the kinesio taping method. Tokyo, Japan: Ken lkai Co. Ltd; 2003. 
14. HM Klein, R Brockmann, September 2015, Pain diminishing effect of kinesio taping in patients after sternotomy, Journal of Cardiothoracic Surgery 2015, 10(Suppl 1):A76.

15. Rodriguez S. Pain measurement in the elderly: a review. Pain Manag Nurs 2001; 2: 38-46.

16. Johnson C. Measuring pain. Visual analog scale versus numeric pain scale: what is the difference? J Chirpor Med 2005; 4: 43-44.

17. Ferraz B, Quaresma R, Aquino R et al., Reliability of pain scales in the assessment of literate and illiterate patients with rheumatoid arthritis. J Rheumatol 1990;17: 1022-1024.

18. Peter A. Stark, Paul S. Myles, 2013, Development and Psychometric evaluation of a postoperative Quality of Recovery score- the QoR-15, The American society of Anesthesiologists, Vol. 118:1332-40.

19. Maruko K. Tokyo, Japan: Kinesio taping association 1999, 70-73.

20. Kase K, Wallis J, Kase T. Clinical Therapeutic Applications of the Kinesio Taping Method. Tokyo, Japan 2003; Ken Ikai Co Ltd.
21. Krajczy $\mathrm{M}$ et al. The influence of Kinesio taping on the effects of physiotherapy in patients after laparoscopic cholecystectomy. Sci world J 2012. Article ID 948282, 5 pages doi:10.1100/2012/948282.

22. Szczegielniak J, Krajczy $M$ et al., Kinesio taping in physiotherapy after abdominal surgery, Medsportpress 2007; 3: 299-307.

23. Thelen M, Stoneman D, Dauber A. The clinical efficacy of kinesio tape for shoulder pain: a randomized, double- blinded, clinical trial. J Orhtop Sports Phys Ther 2008; 7: 389-395.

24. Gonz'alez-Iglesias, Fern'andez-De-LasPe'nas C, Cleland J et al., Short term effects of cervical kinesio taping on pain and cervical range of motion in patients with acute whiplash injury: a randomized clinical trial. J Orthop Sports Phys Ther 2009; 39: 515-521.

How to cite this article: Suthar VD, V. P. Hathila. The effectiveness of Kinesio-taping on pain and quality of life in participants with lower abdominal surgery. Int $J$ Health Sci Res. 2021; 11(11): 171-179. DOI: https://doi.org/10. 52403/ijhsr.20211123 